\title{
Linear Stability Analysis of a Non-premixed Buoyant Jet Flame
}

\author{
Yee Chee See* and Matthias Ihme ${ }^{\dagger}$ \\ Department of Aerospace Engineering \\ University of Michigan, Ann Arbor, MI 48109-2140
}

\begin{abstract}
Non-premixed combustion systems are susceptible to hydrodynamic and diffusivethermal instabilities. These instabilities are of practical interest as they can assist in enhancing scalar mixing and turbulent transition. Linear stability theory has been used in the past to characterize the response of non-premixed flames to perturbations, providing valuable information about spatio-temporal growth-rate and instability behavior. To make this analysis tractable, several modeling assumptions about mean-flow profiles, thermo-viscous-diffusive transport properties, and reaction order are commonly employed. The objective of this work is to systematically investigate effects of these underlying modeling assumptions on the stability behavior in jet diffusion flames. To this end, linear stability theory is combined with a flamelet-formulation, so that all constitutive relations, chemical species, heatrelease and chemical source terms are represented in terms of a mixture fraction and a reaction progress variable. This model is applied to a methane/air jet flame that was investigated by Füri et al. [Proc. Combust. Inst., 29, 1653-1661, 2002]. Using this formulation, the sensitivity of the predicted stability behavior to model assumptions for the mean-flow and the description of transport properties is investigated. Detailed comparisons show that the phase speed is relatively insensitive to model assumptions and agrees well with experimental measurements. However, the growth-rate exhibits a pronounced sensitive to the specification of the meanflow and the thermo-viscous properties. Interestingly, it is also shown that different levels of model approximation can lead to drastic changes in the stability behavior, altering the local stability dynamics from an absolutely to a convectively unstable regime.
\end{abstract}

\section{Introduction}

Current combustion technologies for aviation and transportation systems largely utilize nonpremixed combustion systems. In these systems, heat-release and fuel conversion rely on the rapid mixing between reactants. With increasing interest in developing compact combustion systems having higher power-output and energy densities, more effective mixing strategies become necessary. Thermo-diffusive and hydrodynamic instabilities can hereby assist in improving the mixing rate. Hydrodynamic instabilities are manifested by large-scale vortical structures (KelvinHelmholtz modes) that can favorably alter the flame dynamics, and can trigger transition from laminar to turbulent combustion regimes. Matalon ${ }^{1}$ provided an excellent review about the current knowledge of hydrodynamic and thermo-diffusive instabilities in premixed and diffusion flames.

\footnotetext{
${ }^{*}$ Research Assistant, Department of Aerospace Engineering, University of Michigan, AIAA member
}

${ }^{\dagger}$ Assistant Professor, Department of Aerospace Engineering, University of Michigan, AIAA member 
The stability of buoyant jet diffusion flame is a fundamental problem and considerable contributions have been made over recent past. Most notable are early investigations on flame-flickering of candles. ${ }^{2-4}$ By utilizing Mie-scattering and photographic imaging, early work by Chen el at. ${ }^{3}$ revealed details about the unsteady flame structure. Their experiment showed pronounced vortical roll-up in the core of the jet and large vortical structures in the outer region of the jet. Chan et al. reported that the frequencies of these instabilities were in the range between 10 and $18 \mathrm{~Hz}$. Buckmaster \& Peters ${ }^{5}$ performed linear stability analysis and identified the most unstable modified Kelvin-Helmholtz mode at a frequency of $17 \mathrm{~Hz}$. This result is in agreement with the experiment by Chen el at., which is remarkable considering that their linear analysis was performed at an arbitrary downstream location while neglecting viscous and buoyancy effects.

In addition to identifying the most unstable mode, linear stability analysis can also be used to determine the spatio-temporal behavior of the instabilities. Huerre and Monkewitz ${ }^{6}$ provided an extensive review on global and local behaviors of absolute and convective instabilities in nonreacting flows.

Lingens el al. ${ }^{7}$ investigated instabilities of buoyant jet diffusion flames through spectral measurements and linear stability analysis. They reported the transition of the instability from convectively unstable to absolutely unstable near the burner rim. The presence of this absolutely unstable mode explains why the flickering of a candle is self-excited. Juniper et al. ${ }^{8}$ subjected jet flames to external excitation. They observed that their jet flame largely retains the self-excited mode even under external forcing, suggesting that jet flames exhibit regions of absolute instability.

Because of its geometric simplicity, considerable work has been conducted on reacting shearlayer instabilities. One of the early works on reacting shear layers is by Shin \& Ferziger. ${ }^{9}$ In this work they were able to identify three distinct instability modes, namely a central mode in the middle of the shear layer and two outer modes that originate from both fuel and oxidizer streams. Through inviscid linear analysis, Shin and Ferziger's findings suggest that heat release suppresses the central hydrodynamic mode in a shear layer and amplifies the two outer modes. This work was complemented by Day et al. ${ }^{10}$ who performed comprehensive parametric studies to elucidate effects of compressibility, density ratio, velocity ratio and heat release on the three instability modes. Their calculations showed that compressibility, heat release, and fuel/oxidizer density ratio are more relevant in affecting the flow structure than equivalence ratio and velocity difference between both streams.

To make the stability analysis of chemical reacting flames tractable, several modeling assumptions have been invoked. In particular, the base flow is commonly represented using analytical flow profiles. Often, effects of viscosity are neglected and only the inviscid problem is considered. $^{5,10}$ More detailed investigations introduced viscous-contributions by approximating the viscous-diffusive properties either as constant and linearly-dependent on temperature. The latter assumption is referred to as Chapman's relation. In addition, the description of the reaction chemistry is usually limited to a one-step chemical model, providing only an incomplete description about the structure of non-premixed flames. ${ }^{11}$ Despite these simplifications, results obtained from linear stability analysis are in reasonable agreement with measurements. ${ }^{7,12}$ However, it is currently not clear how these modeling assumptions affect the results and conclusions drawn from these stability investigations.

The objective of this work is to systematically assess critical modeling approximations that are commonly employed in linear stability investigations, namely the description of mean-flow profiles, the functional dependence of constitutive relations on temperature and species composition, and the representation of the reaction chemistry. These individual modeling assumptions are systematically analyzed by considering a jet diffusion flame that was investigated by Füri et al. ${ }^{12}$

The remainder of this paper is organized as follows. The mathematical model is discussed next. The experimental configuration, computational setup, and modeling assumptions are presented in 
Sections III and IV. Results are discussed in Section V and the paper finishes with conclusions.

\section{Mathematical Model}

\section{A. Governing Equations}

The instantaneous conservation equations for mass, momentum, mixture fraction, and progress variable can be written in dimensionless form as:

$$
\begin{aligned}
\frac{\partial \rho}{\partial t}+\nabla \cdot(\rho \mathbf{u}) & =0, \\
\frac{\partial(\rho \mathbf{u})}{\partial t}+\nabla \cdot(\rho \mathbf{u u}) & =-\nabla p+\frac{1}{R e} \nabla \cdot(\overline{\bar{\tau}})-\frac{\rho}{F r^{2}} \hat{\mathbf{j}}, \\
\frac{\partial(\rho Z)}{\partial t}+\nabla \cdot(\rho \mathbf{u} Z) & =\frac{1}{S c R e} \nabla \cdot(v \nabla Z), \\
\frac{\partial(\rho C)}{\partial t}+\nabla \cdot(\rho \mathbf{u} C) & =\frac{1}{S c R e} \nabla \cdot(v \nabla C)+\dot{\Omega}_{C} .
\end{aligned}
$$

where $t$ is the time, $\mathbf{u}$ is the velocity vector, $\rho$ is the density, $p$ is the pressure, $Z$ is the mixture fraction, $C$ is the reaction progress variable, and $\dot{\Omega}_{C}$ is the production rate. The viscous stress tensor is denoted by $\overline{\bar{\tau}}, \hat{\mathbf{j}}$ is the unit-vector pointing in the direction of the gravity, $v$ is the species diffusivity, and $\mu$ is the dynamic viscosity. The following dimensionless variables are used in Eqs. (1):

$$
\begin{aligned}
& p=\frac{p^{*}}{\rho_{0} U_{0}^{2}}, \quad \rho=\frac{\rho^{*}}{\rho_{0}}, \quad \mathbf{u}=\frac{\mathbf{u}^{*}}{U_{0}}, \quad \mu=\frac{\mu^{*}}{\mu_{0}}, \\
& v=\frac{v^{*}}{v_{0}}, \quad p=\frac{p^{*}}{p_{0}}, \quad t=\frac{t^{*} U_{0}}{D_{0}}, \quad \nabla=\nabla^{*} D_{0},
\end{aligned}
$$

where an asterisk denotes a dimensional quantity and the subscript "0" refers to a reference quantity. The relevant non-dimensional groups in equation set (1) are:

$$
R e=\frac{\rho_{0} U_{0} D_{0}}{\mu_{0}}, \quad S c=\frac{\mu_{0}}{v_{0}}, \quad F r=\frac{U_{0}^{2} D_{0}}{g} .
$$

For further reference, Eqs. (1) are written in operator form:

$$
\mathcal{M}(\phi, \xi) \phi=0
$$

where the state variables are denoted by

$$
\phi=(\boldsymbol{u}, p, Z, C)^{T},
$$

and the vector of constitutive relations and chemical source term is denoted by

$$
\boldsymbol{\xi}=\left(\rho, \mu, v, \dot{\Omega}_{C}\right)^{T} .
$$

\section{B. Flamelet Formulation and Flamelet/Progress Variable Model}

In the following, a model for the linear stability analysis is derived that utilizes a flamelet combustion model to fully account for detailed reaction chemistry and complex thermo-viscous transport. The underlying assumption of the flamelet-formulation is the consideration of a diffusion flame as an ensemble of laminar one-dimensional flame-structures. ${ }^{13,14}$ At sufficiently large Damköhler number or sufficiently high activation energy, chemical reactions and heat transfer occur in a thin layer. If 
the characteristic length scale of this layer is smaller than that of the surrounding flow, vortical structures are unable to penetrate the reaction zone and cannot destroy the flame structure. The effect of the velocity field in this so-called flamelet regime results in a deformation and stretching of the flame sheet. With this notion, a flamelet can be considered as a thin reaction zone surrounded by a molecular transport layer, which, in turn, is embedded within an unsteady flow. ${ }^{15}$

The one-dimensional laminar flamelet equations can be derived by introducing a new coordinate system that is locally attached to the surface of stoichiometric mixture. If the direction normal to the flame surface is associated with the mixture fraction, and spatial changes along the other directions are neglected, the following one-dimensional steady-state flamelet equations can be derived: ${ }^{16}$

$$
-\rho \frac{\chi_{Z}}{2} \frac{\partial^{2}\{\boldsymbol{Y}, T\}}{\partial Z^{2}}=\left\{\dot{\boldsymbol{\Omega}}, \dot{\Omega}_{H}\right\}
$$

where $\left\{\dot{\boldsymbol{\Omega}}, \dot{\Omega}_{H}\right\}$ is the source term for all species mass fractions and temperature, denoted by $\boldsymbol{Y}$ and $T$, respectively. The scalar dissipation rate is defined as $\chi_{Z}=2|\alpha \nabla Z|^{2}$.

The solution of the flamelet equations, Eq. (6), can then be parameterized in terms of mixture fraction and scalar dissipation rate. This can be written as:

$$
\psi=\mathcal{E}_{\boldsymbol{\psi}}\left(Z, \chi_{Z}\right)
$$

where $\boldsymbol{\psi}=\left(\boldsymbol{Y}, T, \dot{\boldsymbol{\Omega}}, \dot{\Omega}_{H}, \rho, v, \mu\right)^{T}$ is the vector of all thermo-chemical variables, and $\mathcal{E}_{\boldsymbol{\psi}}$ refers to the steady laminar flamelet library. Since this flamelet parameterization is non-unique a progress variable $C$ is introduced. ${ }^{17,18}$ In this so-called flamelet/progress-variable (FPV) formulation, the vector of thermo-chemical quantities is then uniquely parameterized in terms of $Z$ and $C$, and can be written as:

$$
\psi=\mathcal{F}_{\psi}(Z, C),
$$

where $C$ is defined by a linear combination of major product mass fractions or temperature. Note that $\mathcal{F}_{\boldsymbol{\psi}}$ denotes the FPV-library. In the following, this FPV-library is pre-computed prior to the simulation, and all thermo-chemical quantities $\boldsymbol{\xi}$ that appear in Eqs. (1) are then directly retrieved from this table:

$$
\begin{aligned}
\rho & =\mathcal{F}_{\rho}(Z, C), \\
\mu & =\mathcal{F}_{\mu}(Z, C), \\
v & =\mathcal{F}_{v}(Z, C), \\
\dot{\Omega}_{C} & =\mathcal{F}_{\dot{\Omega}_{C}}(Z, C) .
\end{aligned}
$$

\section{Linear Stability Analysis}

In the linear stability analysis, the state variables in Eqs. (4) are decomposed into a parallel mean flow and a perturbation field:

$$
\phi(x, r, \theta, t)=\bar{\phi}(r)+\phi^{\prime}(x, r, \theta, t)
$$

where $\boldsymbol{x}=(x, r, \theta)^{T}$ denote the spatial directions in cylindrical coordinates, $\boldsymbol{\phi}$ is defined in Eq. (4), the overbar denotes an axis-parallel mean-flow quantity, and $\phi^{\prime}$ is the perturbation field. After introducing a normal mode decomposition, the disturbance-field is written as:

$$
\phi^{\prime}(x, r, \theta, t)=\widehat{\phi}(r) \exp \{i(\omega t-\alpha x-m \theta)\} .
$$

where $\widehat{\phi}$ is the eigenfunction, $\omega$ and $\alpha$ are the complex-valued frequency and axial wave number, and $m$ represents the azimuthal mode. 
In chemically reacting flows, the constitutive state-vector of Eq. (5) is dependent on chemical composition and temperature and exhibits strong non-linearities. To accurately account for these non-linearities in the stability analysis, we utilize the FPV formulation, and expand these quantities around mean mixture fraction $\bar{Z}$ and mean reaction progress variable $\bar{C}$. This can be written as

$$
\begin{aligned}
\boldsymbol{\xi} & =\overline{\boldsymbol{\xi}}+\boldsymbol{\xi}^{\prime} \\
& =\mathcal{F}_{\boldsymbol{\xi}}(\bar{Z}, \bar{C})+\left(\left.\partial_{Z} \mathcal{F}_{\boldsymbol{\xi}}\right|_{(\bar{Z}, \bar{C})} Z^{\prime}+\left.\partial_{C} \mathcal{F}_{\boldsymbol{\xi}}\right|_{(\bar{Z}, \bar{C})} C^{\prime}\right), \\
& =\mathcal{F}_{\boldsymbol{\xi}}(\bar{Z}, \bar{C})+\left(\left.\partial_{Z} \mathcal{F}_{\boldsymbol{\xi}}\right|_{(\bar{Z}, \bar{C})} \widehat{Z}+\left.\partial_{C} \mathcal{F}_{\boldsymbol{\xi}}\right|_{(\bar{Z}, \bar{C})} \widehat{C}\right) \exp \{i(\omega t-\alpha x-m \theta)\}
\end{aligned}
$$

in which the expressions $\partial_{Z} \mathcal{F}_{\boldsymbol{\xi}}$ and $\partial_{C} \mathcal{F}_{\boldsymbol{\xi}}$ are directly evaluated by differentiating the FPV-flamelet library. Special care has to be taken when evaluating the derivatives in flamelet space near the boundaries of the flamelet state-space. Upon introducing this flamelet-expression into Eq. (1) and neglecting higher-order terms, the linearized form of the governing equations can be written as

$$
\mathcal{N}(\bar{\phi}, \boldsymbol{\xi}(\bar{\phi})) \phi^{\prime}=0
$$

After introducing the normal mode decomposition, the resulting equations are dependent on the parameters $\omega, \alpha$ and $m$. In the following, a spatial stability analysis is performed. For this, the frequency $\omega$ is real-valued and specified, and the corresponding complex-valued wave numbers $\alpha$ are evaluated as solution of a generalized eigenvalue problem. The imaginary part of the wave number determines the spatial growth rate of the mode and the phase speed of the mode is evaluated as $c_{\text {phase }}=\omega_{r} / \alpha_{r}$.

The general eigenvalue problem is solved using a collocation method. ${ }^{19}$ In this method, the disturbance state-vector $\widehat{\phi}$ is expanded in polynomial space, and the resulting eigenvalue problem (see Appendix A) is solved using a QZ-algorithm.

To determine whether a spatial mode is absolutely or convectively unstable Briggs' method ${ }^{20}$ is employed. The theory behind Briggs' method is discussed in Ref. 6, and is only briefly summarized. Briggs' method requires first the identification of a pair of spatial modes. For an initial value of $\omega_{i}$, the complex axial wave number of each mode is computed as function of $\omega_{r}$, giving pairwise trajectories in the complex $\alpha$ plane. This procedure is repeated for different values of $\omega_{i}$ until the trajectories of the two modes get pinched at a singular point in the $\alpha$ plane. If the pinch point exists for positive-valued $\omega_{i}$ the instability, which is associated with the pair of modes, is considered absolutely unstable. Otherwise, it is classified as convectively unstable.

\section{Configuration and Computational Setup}

In this paper, we consider a jet-diffusion flame configuration that was experimentally investigated by Füri et al. ${ }^{12}$ The experiment consists of an axisymmetric contoured nozzle of diameter $D_{0}=7.5 \mathrm{~mm}$, supplying methane at atmospheric condition. The nozzle is surrounded by a coflow, providing a uniform oxidizer-stream through a porous sintered metal plate. The velocity ratio between coflow and fuel-stream is reported to be less than $1 \%$. The nozzle rim, separating the jet from the coflow, has a thickness of $0.16 D_{0}$. The operating condition considered in this paper corresponds to the experimental setup "Mix 1" of Füri et al. ${ }^{12}$ For reference, relevant parameters of this configuration are summarized in Tab. 1.

To compute the mean-flow, a detailed three-dimensional simulation of the jet configuration is performed. For this, a computational domain of $15 D_{0} \times 15 D_{0} \times 2 \pi$ in axial, radial, and azimuthal direction is used. The computational grid consists of $190 \times 160 \times 32$ grid points in the three respective directions. The reaction chemistry is described using the detailed GRI-2.11 methane/air reaction mechanism, ${ }^{21}$ consisting of 279 reactions among 49 species. The viscosity and diffusivity 


\begin{tabular}{|cc|}
\hline Nozzle diameter $\left(D_{0}\right):$ & $7.5 \mathrm{~mm}$ \\
Jet exit velocity $\left(U_{0}\right):$ & $3.8 \mathrm{~m} / \mathrm{s}$ \\
Froude number $(F r):$ & 14 \\
Reynolds number $(R e):$ & 1768 \\
Lewis number $(\mathrm{Le}):$ & 1 \\
Schmidt number $(S c):$ & 0.7 \\
Oxidizer composition: & $23 \% \mathrm{O}_{2}, 77 \% \mathrm{~N}_{2}$ \\
Fuel composition: & $100 \% \mathrm{CH}_{4}$ \\
\hline
\end{tabular}

Table 1. Parameters of the jet-diffusion flame configuration.

are computed using a species-averaged formulation ${ }^{22,23}$ that is provided through a CHEMkIN-library interface.

It was found that the jet break-up and flow-field dynamics are highly sensitive to inlet-conditions, and variations of less than $20 \%$ of the axial rms-velocity can lead to a reduction of the jet break-up location by three nozzle diameters. To provide an accurate description of the reactant stream, the inlet conditions for this simulation are obtained by performing a separate pipe-flow computation, in which the velocity field is forced to accurately reproduce the measured mean- and root mean squared (rms) velocity profiles along the radial plane closest to the nozzle exit.

The detailed simulation also allowed to characterize the flame-anchoring mechanism in this flame. From this simulation it was found that the flame stabilizes in the low-strain-region just upstream of the burner rim. A comparison of results from an adiabatic simulation with experimental data also suggests that heat-loss effects at the burner rim become significant: The adiabatic flame-temperature of a methane/air mixture at this operating condition is approximately $2250 \mathrm{~K}$. However, temperature measurements near the burner exit are almost $800 \mathrm{~K}$ lower, which cannot be entirely attributed to strain-rate effects or potential uncertainties in the measurements. To account for the heat-losses to the nozzle wall, a non-adiabatic flamelet model was utilized in this simulation. ${ }^{24}$ In this formulation, the unsteady flamelet-equations are solved by adding a convective heat-sink term to the energy-equation.

Results of the detailed simulation for mean temperature and axial velocity are illustrated in Fig. 1. The solid black line corresponds to the isocontour of stoichiometric mixture fraction. From Fig. 1(a) it can be seen that the temperature near the nozzle is reduced due to wall-heat losses. With increasing downstream distance, the flame recovers and reaches its equilibrium composition. The axial velocity profile, shown in Fig. 1(b), follows reasonable well the weakly non-parallel mean-flow approximation that is employed in the present stability analysis.

\section{Modeling Assumptions}

To systematically elucidate the impact of commonly employed modeling approximations on the jet stability, we consider three different configurations, in which individual assumptions are successively relaxed to introduce increasingly more physics into the formulation. To this end, linear stability analysis at an axial location of $x / D_{0}=0.133$ down-stream of the nozzle is performed, and available experimental data are used for model comparisons.

The first configuration that is considered employs an analytic description of the mean-flow, that follows the formulation given by Füri et al. ${ }^{12}$ Since the functional form of the mean temperature field was not explicitly given in Ref. 12, the radial temperature profile is approximated by a flameletprofile that has identical peak temperature than reported by Füri et al. ${ }^{12}$ Thermoviscous properties 


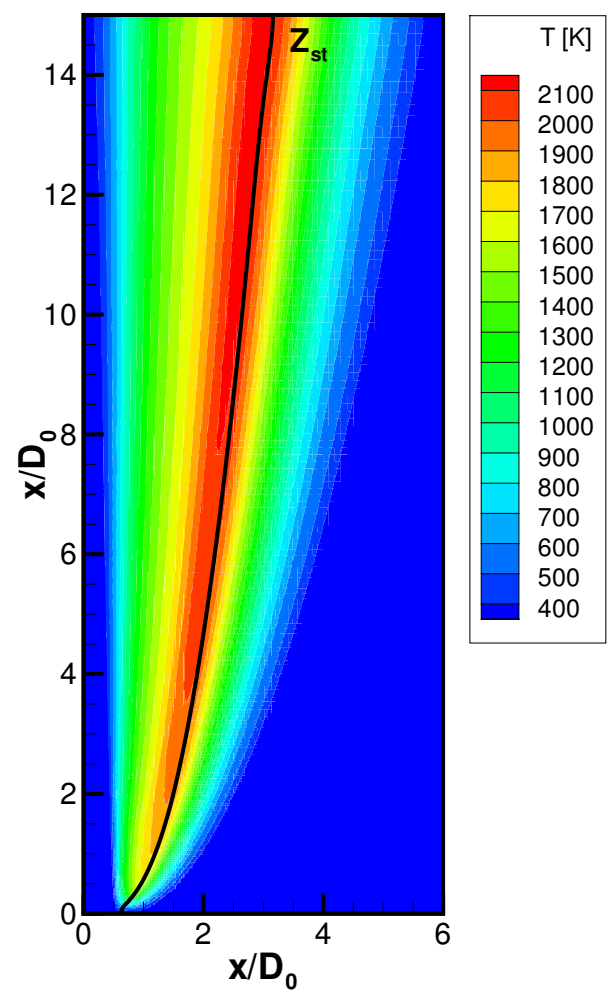

(a) Mean temperature field.

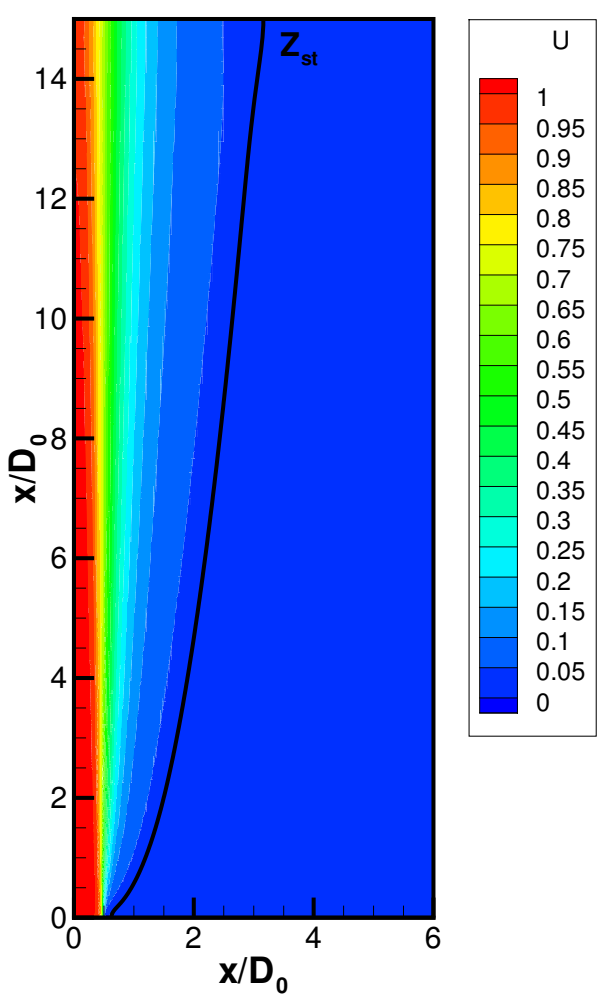

(b) Averaged axial velocity field.

Figure 1. Simulation results for (a) mean temperature and (b) mean axial velocity. The solid line denotes the isocontour of stoichiometric mixture.

in this configuration are described using Chapman's formulation. The configuration that is considered in Case 2 relaxes the description for the thermoviscous properties. In this case, the diffusivity and viscosity are dependent on temperature and species composition using a mixture-averaged formulation. ${ }^{22,23}$ Finally, the third case employs the mean flow profiles for velocity, temperature, and mixture fraction from the detailed simulation and mixture-averaged thermo-viscous properties. For further reference the modeling assumptions that are employed for these three cases are summarized in Tab. 2.

The mean flow profiles for axial velocity and temperature obtained from this simulation are shown in Figs. 2(a) and 2(b), respectively. Apart from a minor shift in the temperature peaklocation, both profiles are fairly similar and in good agreement with the experimental data.

\begin{tabular}{|c|c|c|}
\hline Case & Mean Flow & Thermo-viscous Model \\
\hline 1 & Analytic & Chapman's law \\
2 & Analytic & Mixture-averaged transport \\
3 & Simulation & Mixture-averaged transport \\
\hline
\end{tabular}

Table 2. Summary of investigated configurations and underlying model approximations. 


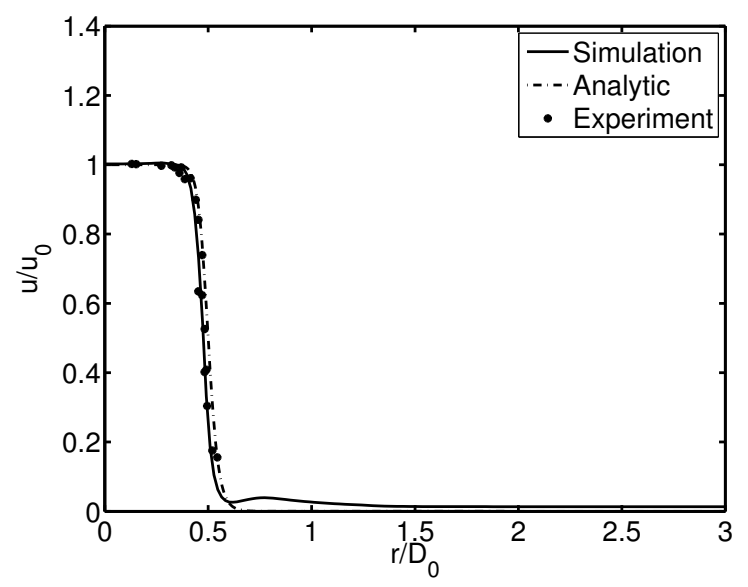

(a) Mean axial velocity.

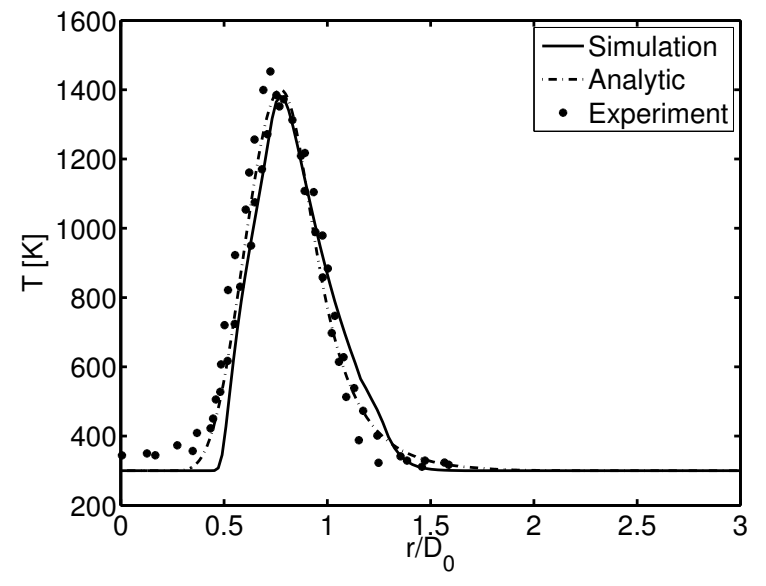

(b) Mean temperature.

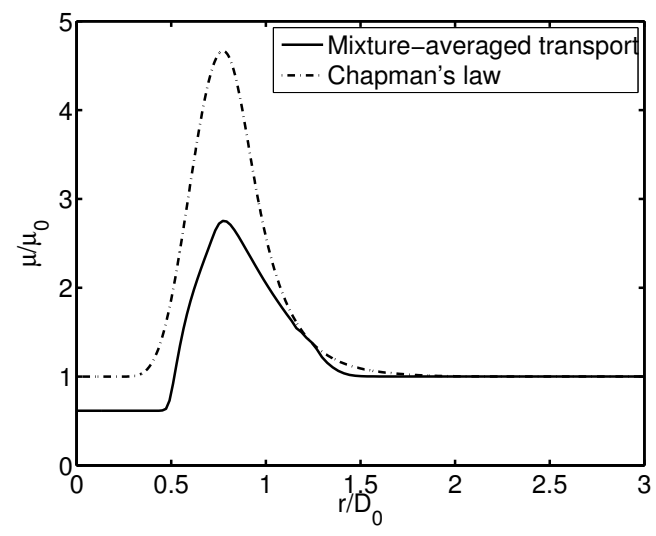

(c) Dynamic viscosity.

Figure 2. Comparison of mean flow profiles at $x / D=0.133$ for (a) axial velocity, (b) temperature, and (c) dynamic viscosity.

\section{Result and Discussion}

In the following, results from linear stability analysis are discussed. In the present investigation, only the axisymmetric mode, corresponding to $m=0$ in Eq. (10), is considered.

\section{A. Thermo-Viscous Effects}

Comparisons of results for growth rate and phase speed for the three investigated configurations are presented in Fig. 3. Overall, the results for growth rate, obtained from the linear stability analysis, are in reasonable agreement with experimental data for frequencies below $\omega=3$. However, with increasing frequency, discrepancies between the three configurations become apparent. Specifically, Fig. 3(a) shows that the predicted growth rate for Case 1 is significantly smaller than predicted by Case 3. This discrepancy can be attributed to the following two reasons. First, the viscousdiffusive properties predicted using Chapman's approximation are significantly higher than the mixture-averaged transport properties. Since previous findings suggest that this instability mode is related to an inviscid Kelvin-Helmholtz mechanism, this mode is damped by viscosity. In fact, an inviscid analysis using the analytical mean-flow profile predicts a growth rate that is approximately twice larger than for Case 3. 


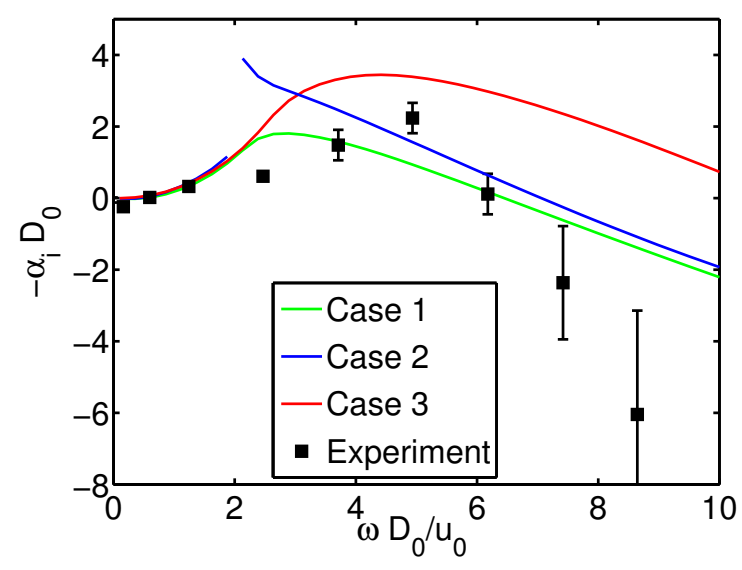

(a) Growth rate.

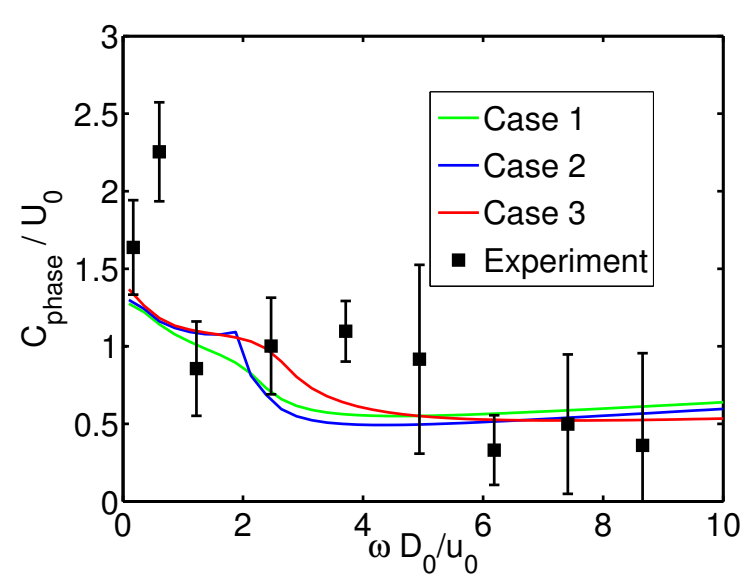

(b) Phase speed.

Figure 3. Comparison of results from linear stability analysis and experiments for (a) growth rate and (b) phase speed at the axial location $x / D_{0}=0.133$.

Figure 3(b) displays a discontinuity in the growth rate for Case 2 around $\omega=2$. At this condition, the mode shown in figure $3(\mathrm{a})$ is switched due to the pinching of two branches. To further substantiate this point, Briggs' analysis is performed to characterize the modal behavior. Results for wave number and frequency at the pinch-point are summarized in Tab. 3 and Fig. 4. For Case 2 the two spatial branches are pinched at $\omega_{\text {pinch }}=2.53+0.063 i$, corresponding to an absolutely unstable mode. In comparison, the pinch point for Case 1 with viscous-diffusive properties described by Chapman's law is at $\omega_{\text {pinch }}=2.41-0.436 i$, and therefore corresponds to a convectively unstable behavior. This suggests that higher values of viscosity move the pinch-point away from the absolutely unstable region towards the lower $\omega$-half-plane.

A comparison of the phase-speed as function of the angular frequency $\omega$ for the three different cases is illustrated in Fig. 3(b). Comparisons with experimental data, shown by symbols, are in reasonable agreement. From this figure it can be seen that the phase speed is relatively insensitive to the model approximation, and minor differences are restricted to the mid-frequency range, $1.5 \leq$ $\omega \leq 4$.

\begin{tabular}{|c|c|c|}
\hline Case & $\alpha_{\text {pinch }}$ & $\omega_{\text {pinch }}$ \\
\hline 1 & $1.95-3.05 i$ & $2.41-0.436 i$ \\
2 & $2.33-2.89 i$ & $2.53+0.063 i$ \\
3 & $1.71-3.43 i$ & $2.61-0.311 i$ \\
\hline
\end{tabular}

Table 3. Comparison of wave number and frequency at the pinch-point for three cases investigated.

\section{B. Mean Flow Effects}

Effects of the mean flow on the predicted jet-stability is elucidated by comparing Cases 2 and 3 . Other than the agreement for lower frequencies, the growth rate evaluated for Case 3 is significantly larger than that predicted with the analytic mean-flow profiles for Case 2 . In addition, the pinch-point analysis also shows that the instability is convectively unstably which is different to the absolutely unstable behavior for the case employing an analytical mean-flow profile. Considering that the analytical mean flow is not substantially different to that predicted by the detailed 


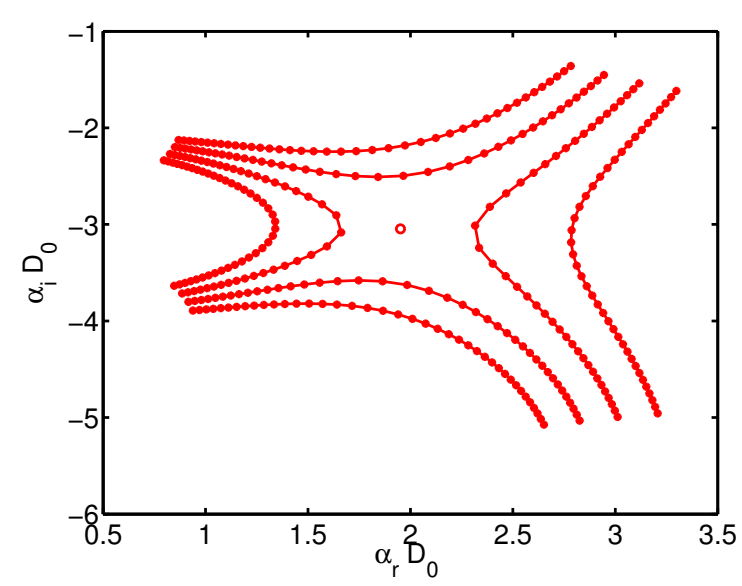

(a) Case 1

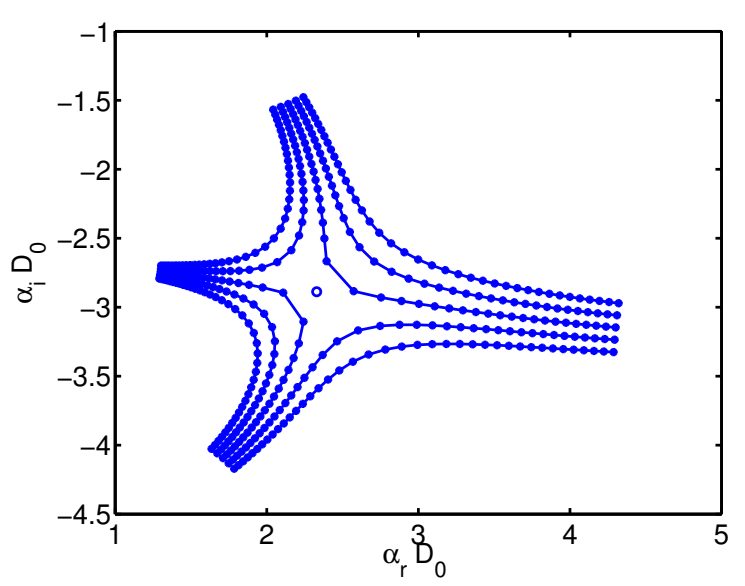

(b) Case 2

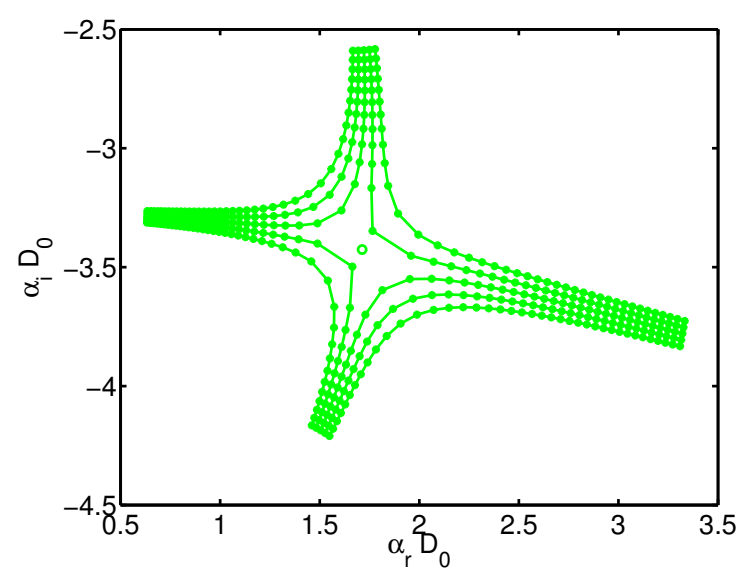

(c) Case 3

Figure 4. Pinch point analysis showing trajectories of wavenumber pairs as function of $\omega=\omega_{r}+i \omega_{i}$.

simulation (see Fig. 2), it can be concluded that the growth rate is highly sensitive to the mean flow.

\section{Conclusions}

Linear stability analysis was combined with a flamelet-formulation to systematically investigate the sensitivity of the predicted stability behavior in a jet diffusion flame to specifications of the mean-flow, thermo-viscous transport properties, and reaction chemistry. The flamelet-formulation represents the structure of a diffusion flame in terms of a reduced set of scalars under consideration of detailed reaction chemistry and complex thermo-viscous-diffusive transport properties.

This model was applied to a jet diffusion flame that was studied by Füri et al. ${ }^{12}$ In the present investigation, three model-configurations were considered: Specifically, the viscous-diffusive transport properties were either represented in terms of Chapman's formulation or by using a mixtureaveraged description accounting for the dependence on temperature and mixture-composition. The second aspect addressed the effect of the mean-flow on the stability behavior. To this end, a detailed simulation was performed, and the mean-flow field was used as input to the stability analysis. The main conclusions from this work are as follows: 
- The growth rate exhibits a sensitivity to the specification of the thermo-viscous-diffusive transport properties. This is mainly attributed to the fact that higher values for viscosity and diffusivity using Chapman's approximation result in a damping of the hydrodynamic instabilities.

- The phase-speed exhibits only modest sensitivity to specification of the mean flow and transport properties, and results are in reasonable agreement with experimental data.

- A comparison of the results from the detailed simulation with experimental data suggests that wall-heat-losses affect the flame structure in the nozzle-near region. To accurately predict the mean flow a non-adiabatic combustion model was employed, providing good agreement of temperature and velocity field with experimental data.

- Although the analytical and computational mean flow profiles are quantitatively very similar, the growth-rate exhibits a pronounced sensitivity to the mean flow specification. This is particularly evident for the configuration employing the highest level of physical complexity.

- Apart from changes in growth-rate, different approximation levels can lead to different instability behaviors, changing the stability dynamics from absolutely to convectively unstable regimes.

\section{Acknowledgment}

The authors gratefully acknowledge financial support through the NSF CAREER program with Award No. CBET-0844587 and the ONR under Grant No. N00014-10-1-0717.

\section{A. Matrix formulation for Generalized Eigenvalue Problem}

The eigenvalue problem that is solved in the linear spatial stability analysis can be written as: is

$$
\mathbf{A} \widehat{\varphi}=\alpha \mathbf{B} \widehat{\varphi}
$$

where the eigenvector $\varphi$ is defined as

$$
\widehat{\varphi}=\left(\hat{u}, \hat{u_{x}}, \hat{v}, \hat{v_{x}}, \hat{w}, \hat{w_{x}}, \hat{p}, \hat{Z}, \hat{Z_{x}}, \hat{C}, \hat{C_{x}}\right)^{T}
$$

The matrix $\mathbf{A}$ is defined as:

$$
\mathbf{A}=\left[\begin{array}{ccccccccccc}
0 & 0 & A_{1,3} & 0 & A_{1,5} & 0 & 0 & A_{1,8} & 0 & A_{1,10} & 0 \\
0 & 1 & 0 & 0 & 0 & 0 & 0 & 0 & 0 & 0 & 0 \\
0 & A_{3,2} & A_{3,3} & A_{3,4} & A_{3,5} & 0 & A_{3,7} & 0 & A_{3,9} & 0 & A_{3,11} \\
0 & 0 & 0 & 1 & 0 & 0 & 0 & 0 & 0 & 0 & 0 \\
0 & A_{5,2} & A_{5,3} & 0 & A_{5,5} & A_{5,6} & A_{5,7} & 0 & 0 & 0 & 0 \\
0 & 0 & 0 & 0 & 0 & 1 & 0 & 0 & 0 & 0 & 0 \\
A_{7,1} & A_{7,2} & A_{7,3} & A_{7,4} & 0 & A_{7,6} & 0 & A_{7,8} & 0 & A_{7,10} & 0 \\
0 & 0 & 0 & 0 & 0 & 0 & 0 & 0 & 1 & 0 & 0 \\
0 & 0 & A_{9,3} & 0 & 0 & 0 & 0 & A_{9,8} & A_{9,9} & A_{9,10} & 0 \\
0 & 0 & 0 & 0 & 0 & 0 & 0 & 0 & 0 & 0 & 1 \\
0 & 0 & A_{11,3} & 0 & 0 & 0 & 0 & A_{11,8} & 0 & A_{11,10} & A_{11,11}
\end{array}\right]
$$


with matrix-components:

$$
\begin{aligned}
& A_{1,3}=-\frac{\bar{\rho}}{r}-\bar{\rho} \frac{\partial}{\partial r}-\frac{\partial \rho}{\partial z} \frac{d}{d r} \bar{z}-\frac{\partial \rho}{\partial c} \frac{d}{d r} \bar{c}, \quad A_{1,5}=\frac{-i \bar{\rho} m}{r}, \\
& A_{1,8}=i \frac{\partial \rho}{\partial z} \omega, \quad A_{1,10}=i \frac{\partial \rho}{\partial c} \omega \\
& A_{3,2}=1 / 3 \frac{\bar{\mu} \frac{\partial}{\partial r}}{R e}-2 / 3 \frac{\frac{d}{d r} \bar{\mu}}{R e} \\
& A_{3,3}=i \bar{\rho} \omega-\frac{\bar{\mu} m^{2}}{R e r^{2}}+4 / 3 \frac{\left(\frac{d}{d r} \bar{\mu}\right) \frac{\partial}{\partial r}}{R e}-2 / 3 \frac{\frac{d}{d r} \bar{\mu}}{\operatorname{Re} r}+4 / 3 \frac{\bar{\mu} \frac{\partial}{\partial r}}{R e r}+4 / 3 \frac{\bar{\mu} \frac{\partial^{2}}{\partial r^{2}}}{R e}-4 / 3 \frac{\bar{\mu}}{R e r^{2}}, \\
& A_{3,4}=-\bar{\rho} \bar{u}, \quad A_{3,5}=\frac{-2 / 3 i\left(\frac{d}{d r} \bar{\mu}\right) m}{\operatorname{Re} r}-\frac{7 / 3 i \bar{\mu} m}{\operatorname{Re} r^{2}}+\frac{1 / 3 i \bar{\mu} \frac{\partial}{\partial r} m}{\operatorname{Re} r} \\
& A_{3,7}=-\frac{\partial}{\partial r} \\
& A_{3,9}=-\frac{1}{R e} \frac{\partial \bar{u}}{\partial r} \frac{\mu}{z}, \quad A_{3,11}=-\frac{1}{R e} \frac{\partial \bar{u}}{\partial r} \frac{\mu}{c} \\
& A_{5,2}=\frac{1 / 3 i \bar{\mu} m}{\operatorname{Rer}}, \quad A_{5,3}=\frac{7 / 3 i \bar{\mu} m}{\operatorname{Re} r^{2}}+\frac{i\left(\frac{d}{d r} \bar{\mu}\right) m}{\operatorname{Re} r}+\frac{1 / 3 i \bar{\mu} \frac{\partial}{\partial r} m}{\operatorname{Re} r} \\
& A_{5,5}=-\frac{\bar{\mu}}{\operatorname{Re} r^{2}}+i \bar{\rho} \omega+\frac{\bar{\mu} \frac{\partial}{\partial r}}{\operatorname{Rer}}-\frac{\frac{d}{d r} \bar{\mu}}{\operatorname{Re} r}+\frac{\bar{\mu} \frac{\partial^{2}}{\partial r^{2}}}{\operatorname{Re}}-4 / 3 \frac{\bar{\mu} m^{2}}{\operatorname{Re} r^{2}}+\frac{\left(\frac{d}{d r} \bar{\mu}\right) \frac{\partial}{\partial r}}{R e} \\
& A_{5,6}=-\bar{\rho} \bar{u}, \quad A_{5,7}=\frac{-i m}{r}, \\
& A_{7,1}=i \bar{\rho} \omega+\frac{\left(\frac{d}{d r} \bar{\mu}\right) \frac{\partial}{\partial r}}{R e}+\frac{\bar{\mu} \frac{\partial^{2}}{\partial r^{2}}}{R e}+\frac{\bar{\mu} \frac{\partial}{\partial r}}{\operatorname{Re} r}-\frac{\bar{\mu} m^{2}}{\operatorname{Re} r^{2}}, \quad A_{7,2}=-\bar{\rho} \bar{u} \\
& A_{7,3}=-\bar{\rho} \frac{d}{d r} \bar{u}, \quad A_{7,4}=1 / 3 \frac{\bar{\mu}}{R e r}+\frac{\frac{d}{d r} \bar{\mu}}{R e}+1 / 3 \frac{\bar{\mu} \frac{\partial}{\partial r}}{R e}, \quad A_{7,6}=\frac{1 / 3 i \bar{\mu} m}{\operatorname{Re} r} \\
& A_{7,8}=-\frac{\frac{\partial \rho}{\partial z}}{F r^{2}}+\frac{1}{R e} \frac{\partial \mu}{\partial z}\left[\frac{\partial \bar{u}}{\partial r}\left(\frac{1}{r}+\frac{\partial}{\partial r}\right)+\frac{\partial^{2} \bar{u}}{\partial r^{2}}\right]+\frac{1}{R e} \frac{\partial \bar{u}}{\partial r} \frac{\partial}{\partial z}\left(\frac{\partial \mu}{\partial r}\right) \\
& A_{7,10}=-\frac{\frac{\partial \rho}{\partial c}}{F r^{2}}+\frac{1}{R e} \frac{\partial \mu}{\partial c}\left[\frac{\partial \bar{u}}{\partial r}\left(\frac{1}{r}+\frac{\partial}{\partial r}\right)+\frac{\partial^{2} \bar{u}}{\partial r^{2}}\right]+\frac{1}{R e} \frac{\partial \bar{u}}{\partial r} \frac{\partial}{\partial c}\left(\frac{\partial \mu}{\partial r}\right) . \\
& A_{9,3}=-\bar{\rho} \frac{d}{d r} \bar{z} \\
& A_{9,8}=i \bar{\rho} \omega-\frac{\bar{v} m^{2}}{\operatorname{Re} S c r^{2}}+\frac{\bar{v} \frac{\partial}{\partial r}}{\operatorname{Re} S c r}+\frac{\left(\frac{d}{d r} \bar{v}\right) \frac{\partial}{\partial r}}{\operatorname{Re} S c}+\frac{\bar{v} \frac{\partial^{2}}{\partial r^{2}}}{\operatorname{Re} S c} \\
& +\frac{1}{R e S c} \frac{\partial \bar{z}}{\partial r} \frac{\partial}{\partial r}\left(\frac{\partial v}{\partial z}\right)+\frac{1}{r \operatorname{Re} S c} \frac{\partial \bar{z}}{\partial r} \frac{\partial v}{\partial z}+\frac{1}{R e S c} \frac{\partial^{2} \bar{z}}{\partial r^{2}} \frac{\partial v}{\partial z}+\frac{1}{R e S c} \frac{\partial \bar{z}}{\partial r} \frac{\partial v}{\partial z} \frac{\partial}{\partial r}
\end{aligned}
$$

$A_{9,9}=-\bar{\rho} \bar{u}, \quad A_{9,10}=\frac{1}{R e S c} \frac{\partial \bar{z}}{\partial r} \frac{\partial}{\partial r}\left(\frac{\partial v}{\partial c}\right)+\frac{1}{r \operatorname{Re} S c} \frac{\partial \bar{z}}{\partial r} \frac{\partial v}{\partial c}+\frac{1}{R e S c} \frac{\partial^{2} \bar{z}}{\partial r^{2}} \frac{\partial v}{\partial c}+\frac{1}{R e S c} \frac{\partial \bar{z}}{\partial r} \frac{\partial v}{\partial c} \frac{\partial}{\partial r}$

$$
A_{11,3}=-\bar{\rho} \frac{d}{d r} \bar{c}
$$

12 of 14 


$$
\begin{aligned}
& A_{11,8}=\frac{\partial \Omega_{c}}{\partial z}+\frac{1}{R e S c} \frac{\partial \bar{c}}{\partial r} \frac{\partial}{\partial r}\left(\frac{\partial v}{\partial z}\right)+\frac{1}{r \operatorname{Re} S c} \frac{\partial \bar{c}}{\partial r} \frac{\partial v}{\partial z}+\frac{1}{R e S c} \frac{\partial^{2} \bar{c}}{\partial r^{2}} \frac{\partial v}{\partial z}+\frac{1}{R e S c} \frac{\partial \bar{c}}{\partial r} \frac{\partial v}{\partial z} \frac{\partial}{\partial r} \\
& A_{11,10}=i \bar{\rho} \omega+\frac{\left(\frac{d}{d r} \bar{v}\right) \frac{\partial}{\partial r}}{\operatorname{Re} S c}+\frac{\partial \Omega_{c}}{\partial c}+\frac{\bar{v} \frac{\partial}{\partial r}}{\operatorname{Re} S c r}+\frac{\bar{v} \frac{\partial^{2}}{\partial r^{2}}}{\operatorname{Re} S c}-\frac{\bar{v} m^{2}}{\operatorname{ReScr^{2}}} \\
& +\frac{1}{R e S c} \frac{\partial \bar{c}}{\partial r} \frac{\partial}{\partial r}\left(\frac{\partial v}{\partial c}\right)+\frac{1}{r \operatorname{Re} S c} \frac{\partial \bar{c}}{\partial r} \frac{\partial v}{\partial c}+\frac{1}{R e S c} \frac{\partial^{2} \bar{c}}{\partial r^{2}} \frac{\partial v}{\partial c}+\frac{1}{R e S c} \frac{\partial \bar{c}}{\partial r} \frac{\partial v}{\partial c} \frac{\partial}{\partial r} \\
& A_{11,11}=-\bar{\rho} \bar{u}
\end{aligned}
$$

The matrix $\mathbf{B}$ is:

$$
\mathbf{B}=\left[\begin{array}{ccccccccccc}
i \rho & 0 & 0 & 0 & 0 & 0 & 0 & B_{1,8} & 0 & B_{1,10} & 0 \\
i & 0 & 0 & 0 & 0 & 0 & 0 & 0 & 0 & 0 & 0 \\
0 & 0 & 0 & B_{3,4} & 0 & 0 & 0 & 0 & 0 & 0 & 0 \\
0 & 0 & i & 0 & 0 & 0 & 0 & 0 & 0 & 0 & 0 \\
0 & 0 & 0 & 0 & 0 & B_{5,6} & 0 & 0 & 0 & 0 & 0 \\
0 & 0 & 0 & 0 & i & 0 & 0 & 0 & 0 & 0 & 0 \\
0 & B_{7,2} & 0 & 0 & 0 & 0 & i & 0 & 0 & 0 & 0 \\
0 & 0 & 0 & 0 & 0 & 0 & 0 & i & 0 & 0 & 0 \\
0 & 0 & 0 & 0 & 0 & 0 & 0 & 0 & B_{9,9} & 0 & 0 \\
0 & 0 & 0 & 0 & 0 & 0 & 0 & 0 & 0 & i & 0 \\
0 & 0 & 0 & 0 & 0 & 0 & 0 & 0 & 0 & 0 & B_{11,11}
\end{array}\right]
$$

and the corresponding matrix-elements are:

$$
\begin{gathered}
B_{1,8}=i \bar{u} \frac{\partial \rho}{\partial z}, \quad B_{1,10}=i \bar{u} \frac{\partial \rho}{\partial c}, \\
B_{3,4}=\frac{-i \mu}{R e}, \\
B_{5,6}=\frac{-i \mu}{R e} \\
B_{7,2}=\frac{-4 / 3 i \mu}{R e} \\
B_{9,9}=\frac{-i v}{R e S c}, \\
B_{11,11}=\frac{-i v}{R e S c},
\end{gathered}
$$

\section{References}

\footnotetext{
${ }^{1}$ Matalon, M., "Intrinsic flame instabilities in premixed and nonpremixed combustion," Annual Review of Fluid Mechanics, Vol. 39, No. 1, 2007, pp. 163-191.

${ }^{2}$ Chamberlin, D. and Rose, A., "The flicker of luminous flames," Proceedings of the Symposium on Combustion, Vol. 1-2, 1948, pp. 27-32.

${ }^{3}$ Chen, L., Seaba, J., Roquemore, W., and Goss, L., "Buoyant diffusion flames," Symposium (International) on Combustion, Vol. 22, No. 1, 1989, pp. 677-684.

${ }^{4}$ Cetegen, B. M. and Dong, Y., "Experiments on the instability modes of buoyant diffusion flames and effects of ambient atmosphere on the instabilities," Experiments in Fluids, Vol. 28, No. 6, June 2000, pp. 546-558.
}

13 of 14 
${ }^{5}$ Buckmaster, J. and Peters, N., "The infinite candle and its stability-A paradigm for flickering diffusion flames," Symposium (International) on Combustion, Vol. 21, No. 1, 1988, pp. 1829-1836.

${ }^{6}$ Huerre, P. and Monkewitz, P. A., "Local and global instabilities in spatially developing flows," Annual Review of Fluid Mechanics, Vol. 22, No. 1, Jan. 1990, pp. 473-537.

${ }^{7}$ Lingens, A., Neemann, K., Meyer, J., and Schreiber, M., "Instability of diffusion flames," Symposium (International) on Combustion, Vol. 26, No. 1, 1996, pp. 1053-1061.

${ }^{8}$ Juniper, M. P., Li, L. K., and Nichols, J. W., "Forcing of self-excited round jet diffusion flames," Proceedings of the Combustion Institute, Vol. 32, No. 1, 2009, pp. 1191-1198.

${ }^{9}$ Shin, D. S. and Ferziger, J. H., "Linear stability of the reacting mixing layer," AIAA Journal, Vol. 29, No. 10, 1991, pp. 1634-1642.

${ }^{10}$ Day, M. J., Reynolds, W. C., and Mansour, N. N., "The structure of the compressible reacting mixing layer: Insights from linear stability analysis," Physics of Fluids, Vol. 10, No. 4, 1998, pp. 993.

${ }^{11}$ Williams, F. A., "Overview of asymptotics for methane flames," Reduced Kinetic Mechanisms and Asymptotic Approximations for Methane-Air Flames, edited by M. D. Smooke, Vol. 384, Springer-Verlag, Berlin/Heidelberg, 1991, pp. 68-85.

${ }^{12}$ Füri, M., Papas, P., Rais, R. M., and Monkewitz, P. A., "The effect of flame position on the Kelvin-Helmholtz instability in non-premixed jet flames," Proceedings of the Combustion Institute, Vol. 29, No. 2, 2002, pp. 1653-1661.

${ }^{13}$ Peters, N., "Local quenching due to flame stretch and non-premixed turbulent combustion," Combust. Sci. Tech., Vol. 30, 1983, pp. 1-17.

${ }^{14}$ Peters, N., "Laminar diffusion flamelet models in non-premixed turbulent combustion," Prog. Energy Combust. Sci., Vol. 10, No. 3, 1984, pp. 319-339.

${ }^{15}$ Williams, F. A., "Descriptions of nonpremixed turbulent combustion," AIAA Paper 2006-1505, 2006.

${ }^{16}$ Peters, N., Turbulent Combustion, Cambridge University Press, Cambridge, 2000.

${ }^{17}$ Pierce, C. D. and Moin, P., "Progress-variable approach for large-eddy simulation of non-premixed turbulent combustion," J. Fluid Mech., Vol. 504, 2004, pp. 73-97.

${ }^{18}$ Ihme, M., Cha, C. M., and Pitsch, H., "Prediction of local extinction and re-ignition effects in non-premixed turbulent combustion using a flamelet/progress variable approach," Proc. Combust. Inst., Vol. 30, 2005 , pp. 793-800.

${ }^{19}$ Schmid, P. J. and Henningson, D. S., Stability and Transition in Shear Flows, Springer, 2001.

${ }^{20}$ Briggs, R., Electron-Stream Interaction with Plasmas, MIT Press, 1964.

${ }^{21}$ Bowman, C. T., Hanson, R. K., Davidson, D. F., Gardiner, W. C., Lissianski, V., Smith, G. P., Golden, D. M., Frenklach, M., and Goldenberg, M., "GRI-Mech 2.11," 1997, available from http://www.me.berkeley.edu/ gri-mech/.

${ }^{22}$ Hirschfelder, J. O., Bird, R. B., and Spotz, E. L., "The transport properties for non-polar gases," J. Chem. Physics, Vol. 16, 1948, pp. 968.

${ }^{23}$ Wilke, C. R., "A viscosity equation for gas mixtures," J. Chem. Physics, Vol. 18, No. 4, 1950, pp. 517.

${ }^{24}$ Lee, D., Thakur, S., Wright, J., Ihme, M., and Shyy, M., "Characterization of Flow Field Structure and Species Composition in a Shear Coaxial Rocket GH2/GO2 Injector: Modeling of Wall Heat Losses," AIAA Paper 2011-6125, 2011 . 